\title{
Redox molecule based SERS sensors
}

\author{
Nicolás G. Tognalli ${ }^{1}$, Pablo Scodeller ${ }^{2}$, Victoria Flexer ${ }^{2}$, Rafael Szamocki², \\ Alejandra Ricci ${ }^{2}$, Mario Tagliazucchi ${ }^{2}$, Ernesto J. Calvo ${ }^{2}$, and Alejandro Fainstein ${ }^{1, \dagger}$ \\ ${ }^{1}$ Centro Atómico Bariloche and Instituto Balseiro, Comisión Nacional de Energía Atómica, \\ 8400 S. C. de Bariloche, Río Negro, Argentina and \\ ${ }^{2}$ INQUIMAE, Facultad de Ciencias Exactas y Naturales, Universidad de Buenos Aires, \\ Pabellón 2, Ciudad UniVersitaria, 1428 Buenos Aires, Argentina
}

\begin{abstract}
We describe a general framework to design nanobiosensors based on a wired enzyme coupled to a redox molecule and integrated with SERS Au core-shell nanoparticles and ordered nanocavities. The response of the proposed sensor is based on the different electronic resonant Raman behavior of the oxidized or reduced electronic states of the molecular wire, and on the surface plasmon amplification induced by the tailored metallic substrate. The nanobiosensors can be interrogated remotely through the resonant Raman scattering intensity recovery or spectral variation of the redox molecule, an Os-complex, when the latter varies its oxidation state. Alternatively, we show through two-color spectro-electrochemistry that Raman scattering is also finely sensitive to oxidation state changes of flavin, a biomimetic system that mimics the active center of many flavoprotein enzymes. We show that multiple sample spectroscopic ellipsometry gives access to the spectral dependence of the optical constants of single redox-molecule layers, and through it to the electronic resonances of the system. All the components for selective molecular recognition and for the generation of an optical amplified signal, are self-contained in the proposed biosensor. As proof of concept a compact SERS sensor responsive to glucose with millimolar concentration in solution is demonstrated.
\end{abstract}

\section{INTRODUCTION}

The ability to sense biological molecules in vitro or in vivo with high amplification while reducing the sample size and with high selectivity has been one of the driving forces of the greatly progressing field of plasmonics. In particular, it has been the motivation behind the enormous developments witnessed by surface enhanced Raman techniques in recent years. We propose for this aim sensors based on two building blocks, that is, on one hand the specific molecular recognition by an enzyme and transfer of charge to a redox wired molecule, and on the other hand the surface enhanced Raman interrogation of the electronic state of the latter.

To put together the interacting system comprised of enzyme and redox molecules, and to accomplish the wiring of the latter to a substrate, different approaches can and have been proposed. A molecular thickness and structure control in the nanometer scale has been demonstrated using layer-by-layer (LbL) deposition by electrostatic adsorption ${ }^{1,2}$. This self assembly method consists of a step-by-step electrostatic adsorption of oppositely charged molecules in solution, with surface charge reversal in every adsorption step. This leads to a repulsion induced restriction to a monolayer in every step. LbL films obtained by these wet chemistry methods are stratified and show interpenetration as revealed by x-ray reflectivity. ${ }^{1}$ This polymer interpenetration of adjacent molecular layers allows efficient "molecular wiring" of biomolecules through redox polyion segmental motion in the selfassembled structure. ${ }^{3}$ Alternatively, the redox molecule can be chemically modified to be directly attached to a metallic substrate, e.g. through a thiol or a grafted benzene group.

Glucose oxidase, an enzyme sensitive to glucose, can be efficiently wired by a redox polymer self-assembled LbL on a thiolated gold surface. ${ }^{4}$ Multilayers of an osmium pyridinebipyridine complex tethered to the linear polymer poly-(allylamine), PAH-Os, and the anionic enzyme glucose oxidase, GOx, deposited alternatingly, were studied by different techniques. ${ }^{5,6}$ The structure and dynamics of the self-assembled redox active polyelectrolyte and enzyme multilayers were investigated by quartz crystal microbalance $(\mathrm{QCM})^{7,8}$ ellipsometry ${ }^{7,8} \mathrm{FTIR}^{9}$ and Raman spectroscopy. ${ }^{10} \mathrm{~A}$ reproducible and linear increase in the film thickness, enzyme loading, and catalytic current with the number of dipping cycles has been found.

These molecular structures can be immobilized either onto flat or nanostructured surfaces, or even on nanoparticles. In fact, core-shell colloidal particles with LbL assembled polyelectrolytes multilayers have been introduced by Caruso and co-workers ${ }^{11-14}$ following the sequential adsorption method developed by Decher for flat surfaces. ${ }^{1}$ Nanoparticles have been reported in different biosensing devices using different transduction methods such as colorimetric, ${ }^{15}$ fluorescent, ${ }^{16}$ electrochemical, ${ }^{17}$ surface plasmon resonance, ${ }^{18}$ magnetic, ${ }^{19}$ and in particular, as is the interest here, surface-enhanced Raman scattering. ${ }^{20-22}$

Different strategies for Raman scattering (SERS) ultrasensitive detection of molecules with complementary performances have been proposed so far. ${ }^{23-29}$ Nanoparticles as described above lead to the largest enhancements with up to single molecule detection sensitivity, but are typically poorly controlled in their structure and performance. ${ }^{30-32}$ For analytical applications, and if a good understanding of the SERS process is desired, it is clear that reproducible, homogeneous and stable-in-time substrates are better adapted for the task. ${ }^{26,27,29}$ One of such substrates, developed a few years ago, is the metal- 
lic nanocavity array system. ${ }^{33-41}$ These substrates, with reported SERS enhancements in the $10^{5}-10^{7}$ range, are reproducible, highly homogeneous, stable, and present a large series of parameters (e.g., the material, and the void diameter and height) that can be experimentally controlled to adapt the plasmon-polariton response to specific aims..$^{40,41}$

In this article we describe the development of molecularly specific biosensors based on the contactless interrogation of redox-molecules by surface enhanced resonant Raman spectroscopy. Different alternatives for the relevant building blocks are addressed. This includes the use of an Os-complex as redox "wiring" molecule. Electrochemical SERS is also illustrated with two colour Raman experiments with tethered flavin molecules on gold as a biomimetic system. The immobilization of these molecules on SERS active substrates is accomplished both by the use of LbL growth or by chemical modification and covalent wiring to the subtrate. We show that the electronic structure of monolayers of the redox-molecules immobilized on substrates can be characterized with variable angle spectroscopic ellipsometry using a multiple sample analysis scheme. This allows to design structures that exploit both the electronic resonant enhancement of Raman signals, and the plasmonic response of specifically engineered SERS substrates. The latter SERS enhancement is demonstrated using ordered nanocavity arrays, or alternatively compact fully integrated sensors with a metallic nanoparticle core. As a proof of concept we put together these blocks to demonstrate a core-shell NP SERS sensor which can discriminate the Os(II) redox state and that reports the concentration of glucose in solution.

The article is organized as follows. In the next section we briefly describe the chemical methods used for the growth of the molecular components and SERS substrates, and we present the experimental apparatus and techniques. The Results and Discussion section that follows is ordered sequentially presenting the different building blocks required to develop a redox-molecule based SERS sensor, and finishes presenting as proof of concept results of glucose sensing based on the proposed technique. In the concluding section results are summarized and prospects of future work are presented.

\section{EXPERIMENTAL}

\section{A. (PAH-Os/PVS $)_{n}$ bilayer growth}

To test the growth, structural quality, electrochemical response, and Raman interrogation of the electonic state of a redox-sensing molecule immobilized through LbL growth, we first describe a study of PAHOs/PVS multilayers (MLs). Here PAH-Os stands for a poly(allylamine) modified with $\mathrm{Os}(\text { byp })_{2} \mathrm{ClPyCHO}$, and PVS for polyvinilsulfonate. The PAH-Os/PVS multilayers were prepared following procedures detailed in previ-

\section{Os bipyridine-pyridine Methylformylizoalloxazine complex}
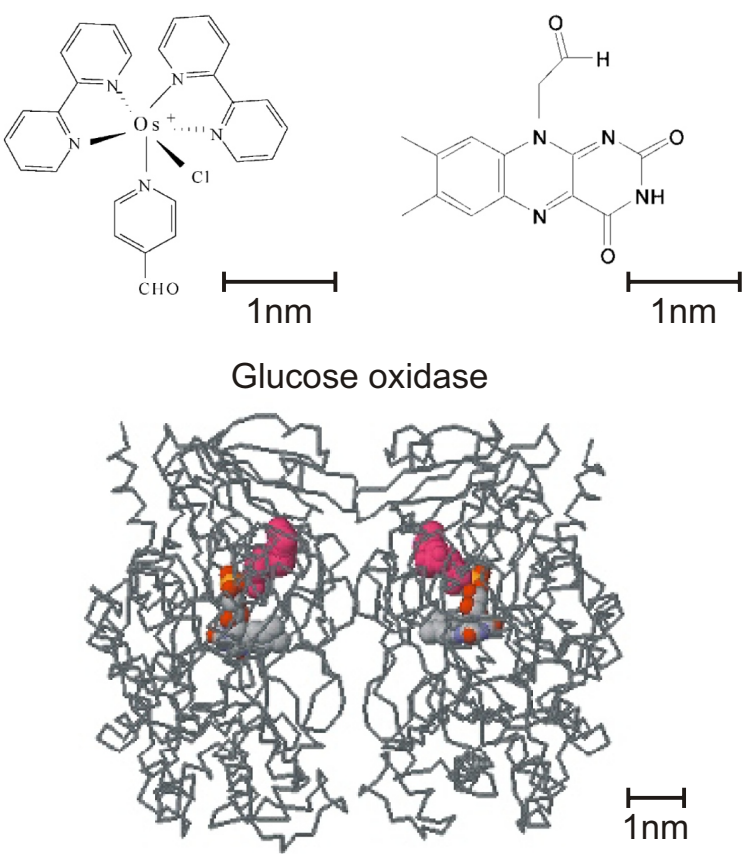

FIG. 1: Top: Scheme of Os bipyridine-pyridine complex (left) and methylformylisoalloxazine (right). Bottom: GOx structure obtained through X-ray diffraction measurements. The globular shape of the macromolecule can be observed with a diameter close to $6 \mathrm{~nm}$. The presence of the two cofactors, The Flavin Adenine Dinucleotide (FAD) is shown in red. Note the difference in size between the redox molecules and the enzyme.

ous publications. ${ }^{9,42,43}$ The substrates, flat gold films $150 \mathrm{~nm}$ thick, were previously modified with 3-mercapto propane sulfonate. The adsorption time for each component, thiol, polymers, and enzyme were determined by a preliminary quartz crystal microbalance study. ${ }^{4}$ The first polycation layer was formed on the thiol-modified $\mathrm{Au}$ substrate by immersion in $\mathrm{PAH}-\mathrm{Os}$ aqueous solution for 10 minutes (see the scheme of the Os complex on the top left corner of Fig. 1). The next and subsequent layers were deposited by alternate immersion in polyanion PVS and polycation aqueous solutions, every step lasting 10 minutes. Each step was completed by thoroughly rinsing with distilled water and drying.

\section{B. Direct bonding of Os complex to Au electrodes}

Osmium bipyridine complex redox molecules were covalently attached to benzoic acid, and through diazonium salt reduction were bonded to $\mathrm{Au}$ electrodes. A glass electrochemical cell with a $\mathrm{Pt}$ counter electrode and a $\mathrm{Ag} / \mathrm{AgCl}, 3 \mathrm{M} \mathrm{KCl}$ reference electrode was employed. Benzoic acid was tethered 


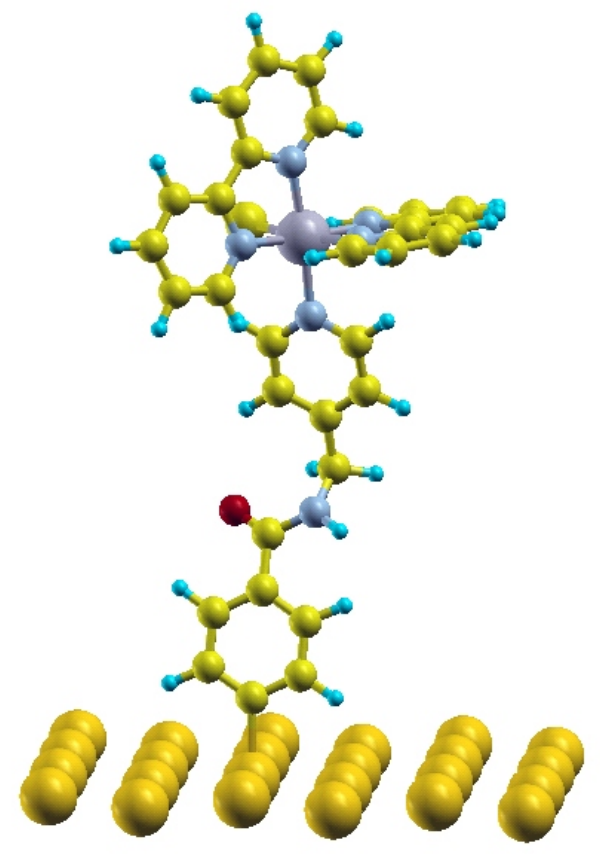

FIG. 2: Scheme of the osmium bipyridine-pyridine chloro complex covalently attached to benzoic acid tethered to $\mathrm{Au}$ by reduction of the diazonium salt of p-mercaptobenzoic acid. $\mathrm{Au}(\mathrm{C})$ is indicated with large(small) yellow symbols, Os with violet, $\mathrm{Cl}$ with red, and nitrogen with indigo. The small light blue symbols correspond to $\mathrm{H}$.

to gold surfaces by electrochemical reduction of the corresponding diazonium salt using chronoamperometry $(0.4 \mathrm{~V}, 5 \mathrm{~min}$ in $5 \mathrm{mM}$ 4-carboxy-phenyl diazonium salt tetrafluoroborate/0.1 M tetrabutylammonium tetra-fluoro borate/acetonitrile). The resulting surface was incubated in $40 \mathrm{mM}$ 1-ethyl-3-(3-dimethylaminopropyl)- carbodiimide/10 mM N-hydroxy succinimide solution for $1 \mathrm{~h}$, and then, it was dipped in 0.25 $\mathrm{mM}\left[\mathrm{Os}(\mathrm{bpy})_{2} \mathrm{Cl}\left(\mathrm{py}-\mathrm{CH}_{2}-\mathrm{NH}_{2}\right)\right]^{+} \mathrm{PF}_{6}^{-} / 0.05 \mathrm{M}$ HEPES buffer (N-2-hydroxyethylpiperazine-N'-2-ethanesulfonic acid) $\mathrm{pH}=7.3, \mathrm{I}=0.1 \mathrm{M} \mathrm{KNO}_{3}$ for $24 \mathrm{~h} .{ }^{44}$ This way, an amide bond was formed between the amino group in the Os complex and the acid group on the surface. The reaction scheme for the posfunctionalization of the bipyridine osmium complex via electroreducion of the diazonium salt of p-mercaptobenzoic acid has been described elsewhere. ${ }^{44}$ A scheme of the resulting modified OS-complex bond to $\mathrm{Au}$ is shown in Fig. 2.

\section{Flavin group attached to a Ag electrode via a Schiff base mechanism}

The flavin group was attached to a nanostructured silver surface using a method previously described in the literature. ${ }^{45}$ The structured silver electrodes were first modified with cysteamine by soaking in a $0.01 \mathrm{M}$ solution in ethanol for $2 \mathrm{~h}$ and then rinsed with ethanol. Methylformylisoalloxazine (top right corner of Fig. 1) was prepared by periodic acid oxidation of riboflavin following a reported procedure. ${ }^{46,47}$ The modified silver electrodes were immersed in $5 \mathrm{~mL}$ of a methanol solution containing $8 \mathrm{mg}$ of methylformylisoalloxazine and $50 \mu \mathrm{L}$ of triethylamine for $12-15 \mathrm{~h}$ in the dark. Excess potassium borohydride was then added to reduce the Schiff base and the solution was cooled in an ice bath and stirred for $1 \mathrm{~h}$ at room temperature. Finally, the electrode was copiously rinsed with water and dried in a jet of nitrogen.

\section{Synthesis of the glucose nanosensor}

\section{Synthesis of Gold Nanoparticles}

Gold nanoparticles were synthetized by a modification of the Turkevich method: In brief, $4.5 \mathrm{mg}$ of tetrachloroauric acid $\left(\mathrm{HAuCl}_{4}\right)$ was dissolved in $45 \mathrm{~mL}$ of MilliQ water, and $10 \mathrm{~mL}$ of this solution was brought to boil in a round-bottom flask under stirring. A $1.6 \mathrm{~mL}$ portion of a $1 \%$ sodium citrate solution and $10 \mu \mathrm{L}$ of $4.2 \mathrm{mg}$ MPS (mercapto propane sulfonate) $/ 100 \mu \mathrm{L}$ water was added simultaneously to the solution using a stirrer. Boiling was maintained for $20 \mathrm{~min}$ after which the heat was removed and stirring was continued for $15 \mathrm{~min}$. The nanoparticles were then filtered through Whatman No.1 filter paper to remove impurities. The nanoparticle suspension was centrifuged to remove excess thiol; the supernatant was removed, and the remaining NPs were redispersed in water.

\section{Synthesis of Core-Shell Nanoparticles}

PAH-Os was employed as redox polyelectrolyte. Repetitive alternate adsorption of PAH-Os and glucose oxidase (GOx, see bottom panel of Fig. 1) on the MPS thiolated Au NP was performed as described previously. ${ }^{48}$ A nanoparticle suspension was added to a $\mathrm{pH}$ 8.75 PAH-Os solution under stirring at room temperature; this solution was left for $20 \mathrm{~min}$, and then the nanoparticles were washed to remove excess PAH-Os. These nanoparticles were then added to a GOx solution in TRIS buffer of $\mathrm{pH}=8$, again under stirring, and at room temperature. The solution was left for $1 \mathrm{~h}$, after which the nanoparticles were again washed to remove excess GOx. These deposition steps were repeated until the desired number of polymer and enzyme layers were deposited. A transmission electronic microscopy image of the fabricated core-shell nanoparticles will be shown below when presenting the glucose biosensors.

The amount of GOx and PAH-Os incorporated in each adsorption step on the nanoparticles has been determined spectrophotometrically as described elsewhere. ${ }^{48}$ The activity of wired GOx has been determined by adsorbing 
the core-shell nanoparticles on a mercaptopropanesulfonate primed $\mathrm{Au}$ surface and following the adsorption process by quartz crystal microbalance. Further, by addition of glucose to the electrolyte we have verified the activity of wired enzyme by the redox polyelectrolye, PAHOs,in the NPs shell (see ref. ${ }^{48}$ for details).

\section{Oxidation of Os(II) Nanoparticles}

The Os(II) bipyridine-pyridine complex cannot be oxidized directly by molecular oxygen or hydrogen peroxide even in the presence of $\mathrm{Au}$ nanoparticles. Therefore, soybean peroxidase (horseradish peroxidase could also be used) was added to the nanoparticle solution, and after incubation for 5 min a $\mathrm{H}_{2} \mathrm{O}_{2}$ solution was added and incubated again for $15 \mathrm{~min}$. Thorough washing cycles were performed to separate the nanoparticles which were then redispersed in water. The oxidation of PAH-Os(II) with $\mathrm{H}_{2} \mathrm{O}_{2}$ catalyzed by soybean peroxidase (SBP) and further reduction with glucose has been followed by UV-visible spectroscopy. ${ }^{48}$

\section{E. Fabrication of metallic nanocavities}

The nanovoid arrays have been fabricated via an hexagonal close packed self-assembly of polystyrene spheres (Duke Scientific Corporation), followed by an electrochemical $\mathrm{Au}$ or $\mathrm{Ag}$ deposition as described by Bartlett and coworkers. ${ }^{33}$ The monolayer self-assembly of polystrene spheres was performed in a thin cell made of a cysteamine modified Au-coated glass slide (AU.1000.ALSI, Platypus Technologies), a clean glass slide, and sidewalls made of a $300 \mu \mathrm{m}$ thick spacer of Parafilm. Spheres are pulled to the evaporation line as the fluid dries, forming a close-packed monolayer domain. The self-assembled polystyrene sphere template is then immersed into an electrochemical plating bath. By controlling the deposition rate and time the cavity height can be finely determined. Finally, the sample is sonicated in dimethylformamide (DMF) to remove the polystyrene template. Very clean, reproducible, and welldefined hexagonal arrays of $\mathrm{Au}$ or $\mathrm{Ag}$ nanocavities are obtained through this method over areas of $\sim 1 \mathrm{~cm}^{2}$.

By systematically retracting the sample from the electrochemical bath during the metal deposition, it is possible to achieve different cavity heights in one single graded sample. In Fig.3 we present typical scanning electron microscopy (SEM) images of cavity arrays with void diameters between 500 and $900 \mathrm{~nm}$ (from top to bottom) and presenting different cavity heights ranging from $h \sim 0.1$ (very shallow) on the left to $h \sim 0.9$ (almost closed) on the right. Here $h$ is given as a fraction of void diameter. The sample quality is evident in these figures, particularly for the $900 \mathrm{~nm}$ voids which are almost perfect for all void heights. Note, however, that some amount of defects develop for the smaller cavities and with thicker structures.

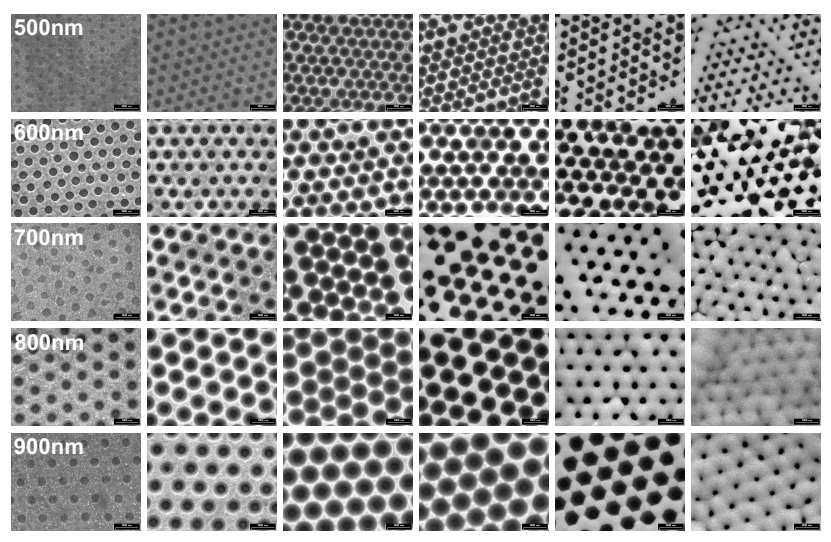

FIG. 3: SEM images taken from five different graded cavity samples with void diameters ranging from 500 to $900 \mathrm{~nm}$ (from top to bottom, indicated in the first figure of each row). The void height go from $h \sim 0.1$ to $h \sim 0.9$ when we move from left to right along each row.

\section{F. Raman experiments}

The resonant Raman scattering experiments were performed using a Jobin-Yvon T64000 triple spectrometer equipped with a liquid- $\mathrm{N}_{2}$ cooled charge coupled device (CCD). The excitation was done using energies between $1.916 \mathrm{eV}(647 \mathrm{~nm})$ and $2.707 \mathrm{eV}$ (458 nm) from an Ar-Kr ion laser. Typical powers ranged from 1-100 mW, concentrated typically in a $20 \mu \mathrm{m}$ diameter spot. To avoid accumulating any possible photobleaching, a fresh spot in the sample was used for every new spectra. For the resonant Raman scans the Raman intensity was corrected for the set-up response (gratings plus CCD).

Raman experiments were also performed during in-situ electrochemical cycles. For this purpose a three electrode cylindrical spectroelectrochemical cell with a spectrosil quartz window was employed. The modified electrodes were placed in front of the cell window under laser illumination. Outside the light path a $1 \mathrm{~cm}^{2}$ platinum mesh was used as auxiliary electrode, and a $\mathrm{Ag} / \mathrm{AgCl}$ reference electrode in $3 \mathrm{M} \mathrm{KCl}$ was employed. A purpose-built operational amplifier potentiostat was employed to control the applied potential of the working electrode.

\section{G. Optical absorption and spectroscopic ellipsometry}

Optical transmission experiments were performed at room temperature using a double-beam UV-Vis Shimadzu UV-1606 spectrophotometer. 
The ellipsometric measurements on the (PAHOs/PVS $)_{n}$ bilayers were performed using a fully automated Wollam WVASE32 variable angle spectroscopic ellipsometer. The instrument software allows multiple sample data analysis and modelling using multiple oscillators.

\section{H. SEM and TEM measurements}

The SEM images were taken with a Phillips XL30 microscope equipped with a $\mathrm{LaB}_{6}$ filament operating at $30 \mathrm{keV}$.

A Philips CM 200 TEM transmission microscope equipped with an ultrathin objective lens was used and the acceleration voltage was $200 \mathrm{keV}$. For TEM observations the core-shell Au nanoparticles were deposited on ultrathin commercial carbon support films deposited on $\mathrm{Cu}$ grids. The thickness of the carbon film was approximately $4 \mathrm{~nm}$.

\section{RESULTS AND DISCUSSION}

\section{A. Ellipsometric study of PAH-Os/PVS MLs}

We begin this section presenting a spectroscopic ellipsometry study of ultrathin molecular PAH-Os/PVS multilayers. The purpose of this study is to determine the film thickness as a signature of the multilayer structure. Through the determination of the complex dielectric function we also access the electronic structure of the redox molecules that are immobilized on the substrate as part of the multilayer. This electronic structure is relevant for the design of the SERS substrate so that the structure performs both as an electronic and plasmonic resonant Raman sensor.

Fig. 4 presents in its top and middle panels the real and imaginary part of the refractive index of PAH-Os/PVS $)_{n}$ MLs derived from a spectroscopic ellipsometry study in dry enviroment of structures with $n=1,3,5,10$ and 15 . Such studies are not straightforward when, as is our case, the optical constants $n$ and $k$ are not known, the film thickness is also unknown, and the latter corresponds to an ultrathin layers of thickness $l \ll \lambda$. Here $\lambda$ is the wavelength of light. The situation is particularly critical when $l<20 \mathrm{~nm}$, as is our case, because of the large correlations existent between the unknown parameters, leading to large uncertainties in the treatment of the ellipsometric data. The data shown in the top and middle panels of Fig. 4 were derived using a multiple sample analysis with a Gauss oscillator model of the variable angle spectroscopic ellipsometry data as described in detail in the Supplementary Information. ${ }^{49}$ Fig. 4 presents the curves corresponding to the Gauss oscillator model (solid lines), and the values of $n$ and $k$ obtained "point by point" using the film thicknesses $l$ obtained with the multiple sample analysis. The agreement between the two approaches

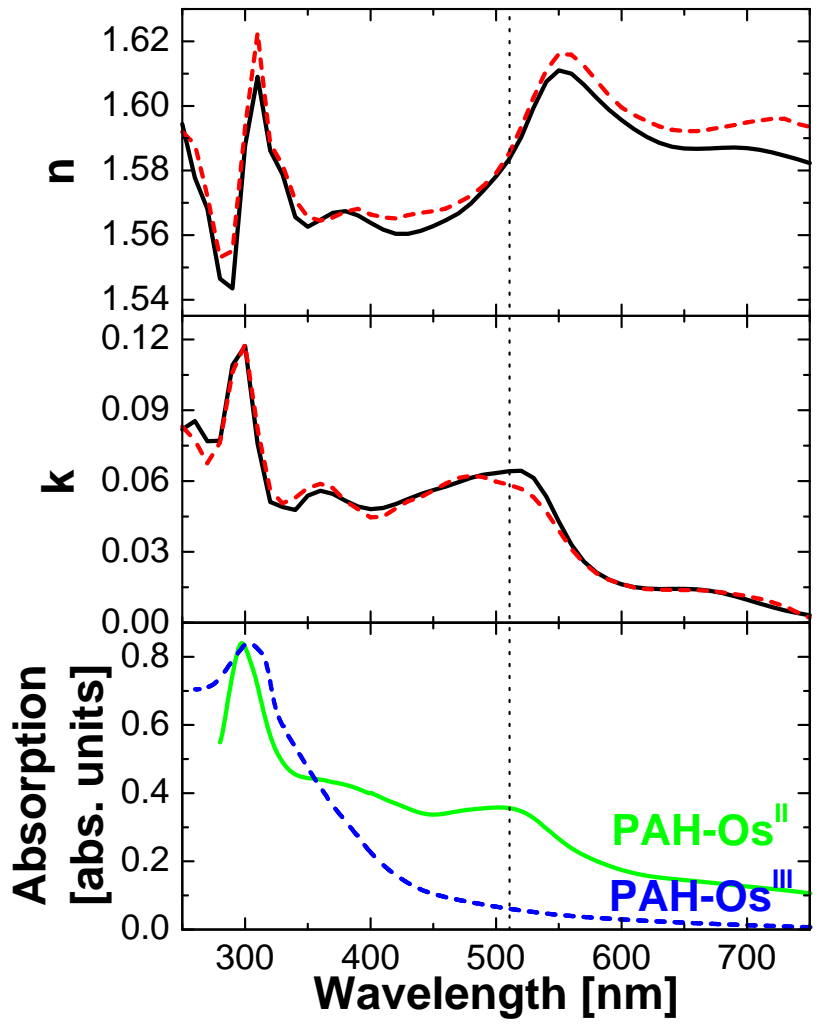

FIG. 4: Variable angle spectroscopic ellipsometry measurements derived from data taken on five (PAH-Os/PVS $)_{n}(n=1$, $3,5,10$ y 15 ) multilayers processed using a Woollam multiple sample analysis software. The top (middle) panel presents the real (imaginary) part of the refraction index, $n(k)$, in the UV-Vis spectral region obtained through a point by point experimental adjustment (dashed red curve) and using a multiple Gauss oscillator model (full black curve). The bottom panel presents a comparison of the optical absorption spectra for PAH-Os in solution both reduced (Os ${ }^{I I}$, green full curve) and oxidized with ceric ions (PAH-Os ${ }^{I I}$, blue dashed curve). Note the disappearance of the absorption at $\sim 510 \mathrm{~nm}$ indicated by the dotted line. The latter is related to the MLCT transition that shifts into the UV region for the Os(III) state.

to derive the wavelength dependent optical constants is quite noteworthy, thus providing an additional sign of consistency of the model used. The real part of the refractive index $n$ varies around 1.58, a value that is also consistent with data obtained in similar molecular systems. ${ }^{43,50}$

Fig. 4 also presents in its bottom panel absorption measurements performed for the PAH-Os complex in solution (solid curve labeled as PAH-OS ${ }^{I I}$ ). We note that PVS has optical transitions only in the UV, and thus all the absorption in the visible region can be assigned unambiguously to the PAH-Os complex. It is worth noting the similarity of the PAH-Os absorption data with the values of $k$ derived from the ellipsometric measurements 
obtained from only a few molecular layers. This gives further support to the procedure followed, and indicates that the molecular structure of PAH-Os is conserved in the multilayer. The peak centered at $\sim 510 \mathrm{~nm}$ is related to the metal-to-ligand charge transfer (MLCT) transition of the Os-complex, and will be particularly relevant in the resonant Raman scattering experiments described below.

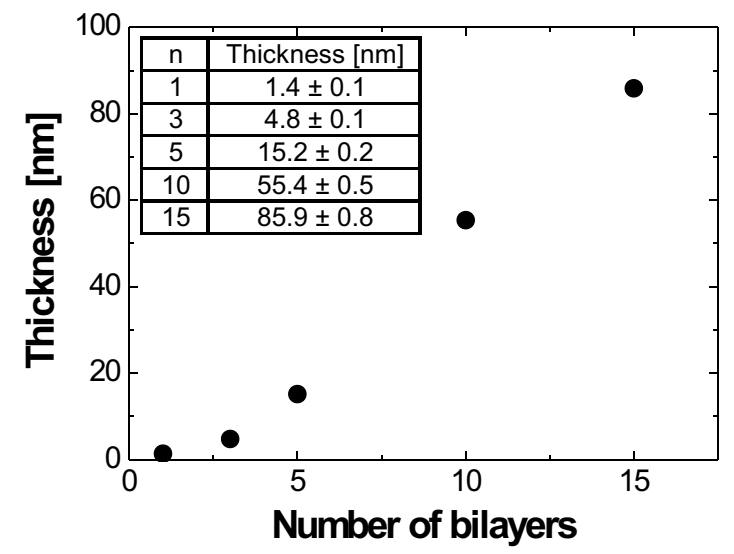

FIG. 5: (PAH-Os/PVS $)_{n}$ multilayer thicknesses as a function of $n$ derived from the multiple sample ellispometric study. The table in the inset gives the values obtained with their experimental uncertainty.

Fig. 5 presents the thickness obtained from the VASE measurements for the (PAH-Os/PVS $)_{n}$ multilayers with $n=1,3,5,10$ and 15 . These values are also given with their experimental error in an inset. The thickness follows a linear behavior for $n \geq 3$, but clearly departs from linearity for the deposition of the first bilayers. This result is consistent with quartz crystal microbalance experiments performed in the same system. ${ }^{43}$

\section{B. Electronic resonant Raman scattering of PAH-Os/PVS MLs}

In the previous section we have shown that PAH-Os assembles in LbL grown PAH-Os/PVS multilayers conserving the molecular structure, displaying a MLCT transition at $\sim 510 \mathrm{~nm}$, and with a thickness that grows approximately linearly with the number of bilayers except for the first couple of deposition steps. We will see in this section that the observed MLCT electronic transition can be used to enhance the Raman signal allowing a spectroscopic study of single molecular bilayers.

Fig. 6 presents spectra of a $(\mathrm{PAH}-\mathrm{Os} / \mathrm{PVS})_{1}$ bilayer for varying laser excitation ranging from $647 \mathrm{~nm}$ (red) to $457 \mathrm{~nm}$ (violet). The spectra displayed correspond to the raw data uncorrected for the spectrometer response. Note that the observed Raman peaks are strongly amplified around $514 \mathrm{~nm}$, and become almost unobservable in

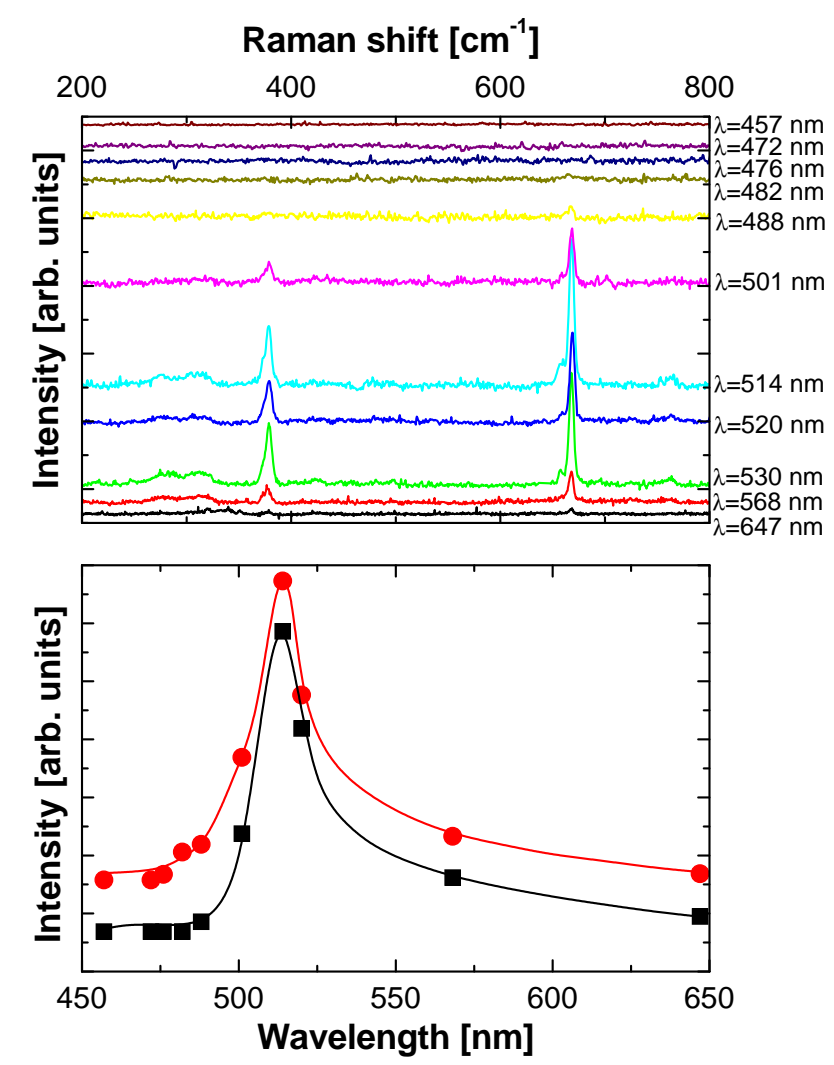

FIG. 6: Top: Raman spectra, taken at $80 \mathrm{~K}$, for a (PAHOs/PVS $)_{1}$ bilayer with varying laser excitation ranging from $647 \mathrm{~nm}$ (red) to $457 \mathrm{~nm}$ (violet). The spectra displayed correspond to the raw data uncorrected for the spectrometer response. Bottom: Raman intensity as a function of laser energy for some of the most intense peaks: $383 \mathrm{~cm}^{-1}$ (squares) and $670 \mathrm{~cm}^{-1}$ (circles). The curves are guides to the eye.

the red and in the violet. The two main peaks that appear in the displayed low energy range are related to the metal or the ligand pyridines and bipyridines (see the scheme in Fig. 1), and can be assigned by comparison with similar molecules. ${ }^{51,52}$ The peak at $\sim 383 \mathrm{~cm}^{-1}$ is associated to a vibration involving the Os- $\mathrm{N}$ bond, while the line at $\sim 670 \mathrm{~cm}^{-1}$ can be related to a pyridine bending mode. The intensity of these two modes as a function of laser wavelength, corrected for the set-up response, is also shown in the bottom panel of Fig. 6. As is clear from this figure the resonant excitation with the MLCT transition leads to a selective amplification of the vibrations related to the metal center (Os and ligand). ${ }^{53,54}$ The resonance is strong enough to allow the observation of a single PAH-Os monolayer with good signal-to-noise ratio. 


\section{Raman determination of Os[II $]$ vs. Os[III $]$} oxidation state

Once we have established that a Raman experiment resonant with the MLCT transition is sensitive to vibrations involving the Os-center in PAH-Os, we wonder whether such an experiment can be also sensitive to molecular changes associated to an oxidation-reduction modification of the molecule.

For this purpose we display in Fig. 7 resonant Raman spectra acquired on a (PAH-Os/PVS $)_{11}$ ML in-situ during an oxidation-reduction cycle. We center our attention in the region around $1550 \mathrm{~cm}^{-1}$ where pyridinestretching vibrations can be identified. The full cycle, from $0 \mathrm{mV}$ to $510 \mathrm{mV}$ and back again to $0 \mathrm{mV}$ was scanned at $2 \mathrm{mV} / \mathrm{s}$, and each spectrum was integrated for 5 seconds. The $514.5 \mathrm{~nm}$ laser power was $10 \mathrm{~mW}$. A $0.1 \mathrm{M}$ acetic acid/sodium acetate buffer solution of $\mathrm{pH} 4.5$ and $0.5 \mathrm{M}$ ionic strength with $\mathrm{KNO}_{3}$ was used as supporting electrolyte. It is quite clear that the most intense lines at $\sim 1483 \mathrm{~cm}^{-1}$ and $\sim 1560 \mathrm{~cm}^{-1}$ completely dissapear upon oxidation with applied voltage. A careful observation of the spectra also shows that at the highest applied voltages an additional but much weaker line can be observed to appear at $\sim 1493 \mathrm{~cm}^{-1}$. To make this clearer the top panel of Fig. 7 includes intercalated spectra which were aquired with much longer times (120 s) but with the potential fixed at $0 \mathrm{mV}$ and $500 \mathrm{mV}$. This line at $\sim 1493 \mathrm{~cm}^{-1}$ can be identified with a pyridine stretching vibration with Os in its Os(III) oxidized state.

In the bottom panel of Fig. 7 we display the intensity of the $\sim 1483 \mathrm{~cm}^{-1}(\mathrm{Os}(\mathrm{II}))$ and $\sim 1493 \mathrm{~cm}^{-1}$ $(\mathrm{Os}(\mathrm{III}))$ modes as a function of applied voltage in the electrochemical cell. The data correspond to the Raman data in the top panel. To identify the oxidation state of the structure we also show the voltamperometric response measured simultaneous to the Raman spectra. The $\sim 1483 \mathrm{~cm}^{-1}$ Raman line disappears when the sample is fully oxidized, and reappears when the reduction process develops. Moreover, the observation of the weaker $\sim 1493 \mathrm{~cm}^{-1}$ peak is complementary to that at $\sim 1483 \mathrm{~cm}^{-1}$, further supporting that it belongs to the Os(III) state of the PAH-Os molecule. Note that within experimental uncertainty the $1483 \mathrm{~cm}^{-1}$ Raman signal is observed to fully dissapear in the oxidized state, indicating a good electronic "wiring" of the Os-complex to the flat $\mathrm{Au}$ substrate.

In order to better understand the resonant Raman scattering results we have performed complementary optical transmission experiments. These are shown in the bottom panel of Fig. 4 where we compare the absorption curve for PAH-Os in solution, with PAH-Os both reduced $(\mathrm{Os}(\mathrm{II}))$ and oxidized with ceric ions (Os(III)). Two features should be highlighted in these curves. First, and as commented above, an intense absorption is observed around $510 \mathrm{~nm}$ in the reduced state. As commented above, this absorption which is responsible for the observed Raman resonance, can be assigned to an

\section{Raman shift $\left[\mathrm{cm}^{-1}\right]$}
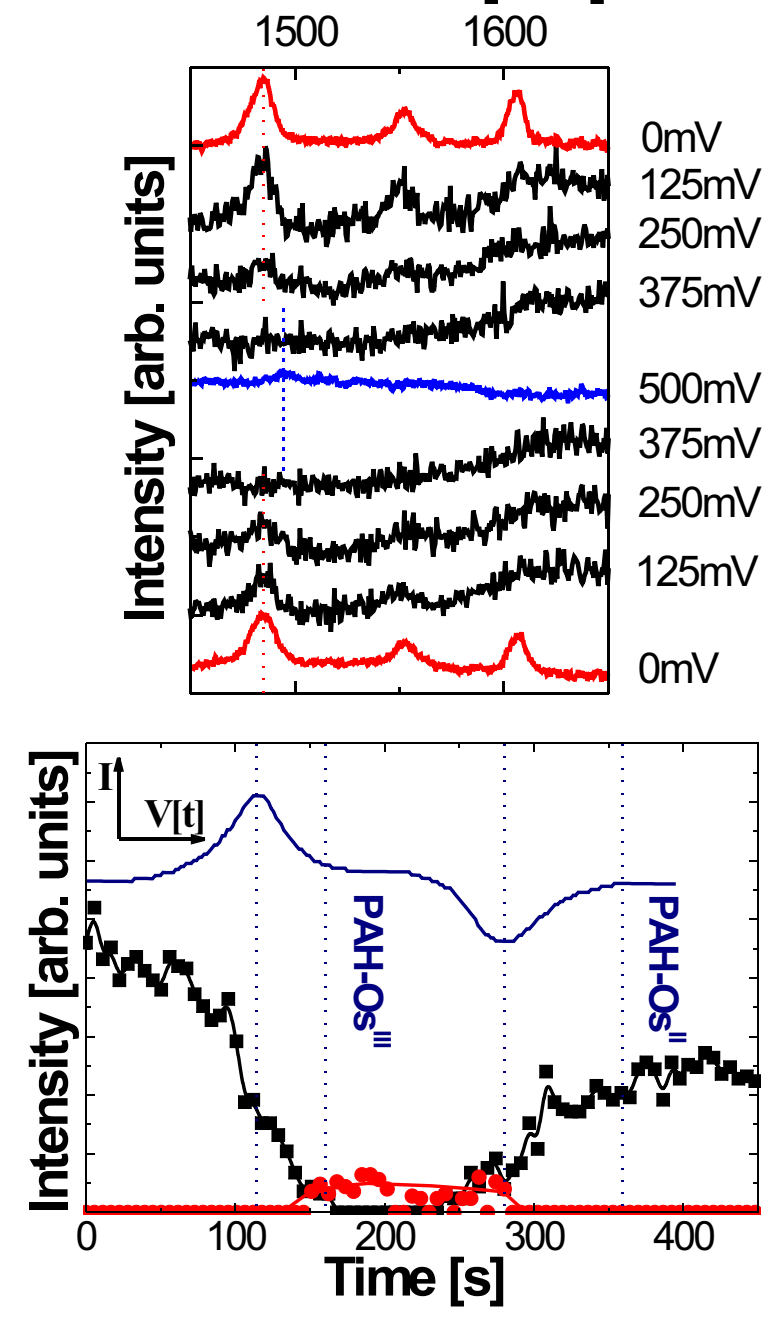

FIG. 7: Top: Evolution of the Raman spectra at some specific potential (presented at the right margin) corresponding to the voltamperometric cycle described in the bottom panel. The red dotted lines highlight the $\sim 1483 \mathrm{~cm}^{-1}$ mode while the blue dashed line indicates the $\sim 1493 \mathrm{~cm}^{-1}$ position. Spectra taken with with longer integration times for fixed potentials 100 and $500 \mathrm{mV}$ are also shown with different colors. Bottom: Intensity of the $\sim 1483 \mathrm{~cm}^{-1}$ (Os(II), full squares) and $\sim 1493 \mathrm{~cm}^{-1}$ (Os(III, full circles) modes as a function of applied voltage measured in situ during an amperometric cycle. The data correspond to a (PAH-Os/PVS $)_{11}$ multilayer and were taken in resonance with the $514.5 \mathrm{~nm}$ laser line. To identify the oxidation state of the structure we also show the voltamperometric response measured together with the Raman spectra. The dotted curves are guides to the eye.

MLCT transition. Second, while the deep UV electronic transitions remain for both complexes, the $\sim 510$ peak disappears in the oxidized state. This confirms its assignment to an MLCT transition from the fully occupied $t_{2 g}$ electronic state of the metal to the ligand LUMO levels. 
In fact, the metal states are expected to decrease in energy in the oxidized state due to the reduced screening, thus strongly shifting the MLCT transition to higher energies. The disappearance of the MLCT absorption at $\sim 510 \mathrm{~nm}$ thus explains the observed quenching of the Raman resonance in the oxidized state.

\section{Spectro electrochemistry of osmium complex covalently tethered to Au-nanocavities}

In the previous section we have shown that we can identify the oxidation-reduction state of the Os-complex in PAH-Os immobilized by electrostatic forces in PAHOs/PVS multilayers. While we showed that resonant Raman spectra can be detected from single bilayer structures on polished $\mathrm{Au}$ surfaces, in a voltammetric cycle where spectra have to be taken with short acquisition times we had to rely on 11 bilayers to obtain data with reasonable signal-to-noise ratio. The aim of the present section is to develop and study an alternative way to immobilize PAH-Os, which is highly stable and is efficiently connected electronically to a metallic substrate. For this purpose we have modified the Os-complex so that it can be covalently attached to $\mathrm{Au}$ or Ag through an aromatic molecule. The molecule-metal covalent bond provides the required stability, while the aromatic ring with a delocalized charge is introduced to improve the "wiring" of the Os-redox center to the metal. The immobilization method, however, does not lead to a full monolayer coverage. STM images indicate in fact a density of $\sim 8 \cdot 10^{4}$ Os-complexes $/ \mu m^{2}$. For this reason, we require a Raman amplification scheme if the electrochemical response of the redox-molecule is to be probed in-situ. For this purpose, to detect and follow the electrochemical behavior with Raman scattering, we will rely on SERS amplification using Au-nanocavities with plasmon excitation tailored to coincide with the electronic resonance of PAH-Os.

We have chosen to use as substrate $\mathrm{Au}$ nanocavity arrays that have shown to provide strong enhancements with good homogeinity and reproducibility. Metallic nanocavity arrays, developed by Bartlett and coworkers, ${ }^{33}$ present two different families of surface plasmon polaritons (SPPs): Mie or localized SPPs (LSPPs), and Bragg or propagating SPPs (PSPPs). ${ }^{55}$ LSPPs correspond to the modes of a cavity in an infinite metallic medium $^{34,56}$, while PSPPs are propagating modes modified by the rupture of the full translational invariance of a metallic surface caused by a periodic corrugation. In real structures, were the voids are truncated and LSPPs and PSPPs coexist, the modes couple and display a complex behavior as a function of cavity diameter, void-height, and angle. This behavior, however, can be quite well understood and theoretically predicted. ${ }^{36-38}$

Based on these considerations we worked with $600 \mathrm{~nm}$ diameter Au cavity-arrays with void height $h=0.5$ that display a ${ }^{1} D$-symmetry plasmon at $\sim 520 \mathrm{~nm}$, well tuned
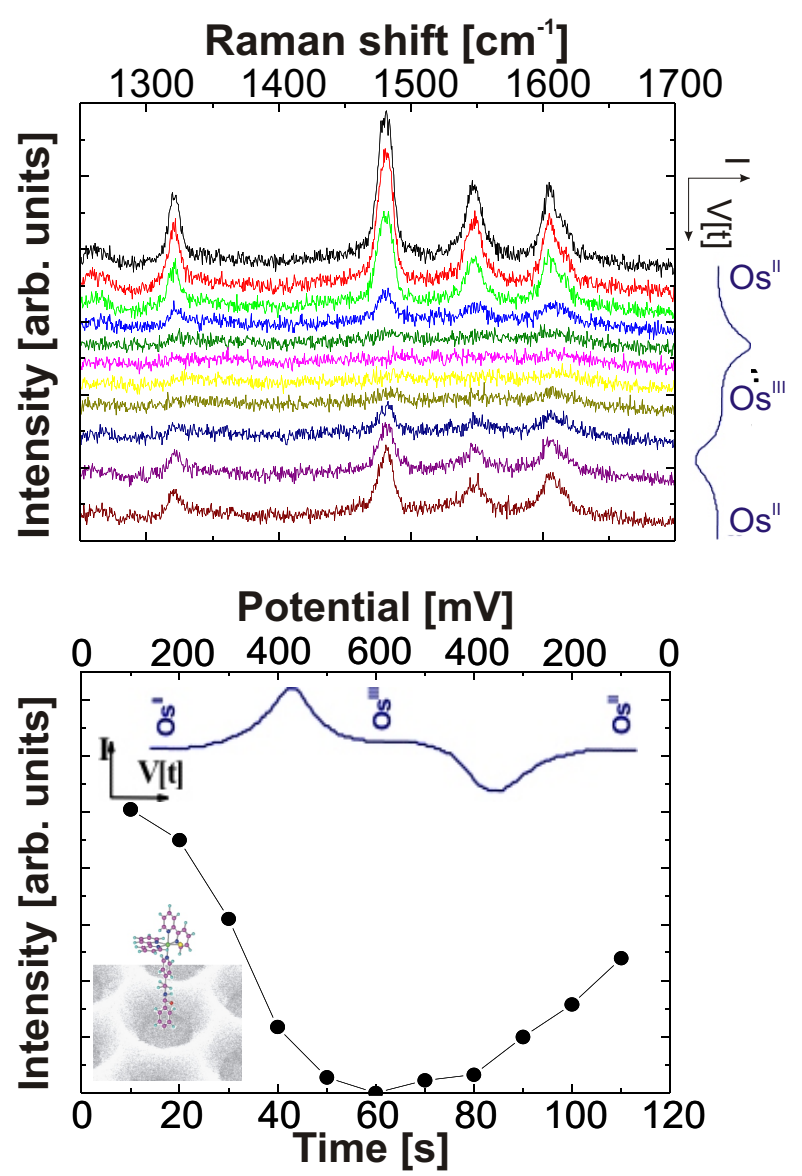

FIG. 8: Top: Raman spectra measured with the $514.5 \mathrm{~nm}$ laser line, using an acquisition time of $10 \mathrm{~s}$ and a power of $10 \mathrm{~mW}$ as a function of the applied electrode potential between $100 \rightarrow 600 \rightarrow 100 \mathrm{mV}$ vs. $\mathrm{Ag} / \mathrm{AgCl}$ at a $10 \mathrm{mV} / \mathrm{s}$ scan rate Bottom: $1483 \mathrm{~cm}^{-1}$ peak intensity as a function of the applied potential (top axis) or time (bottom axis). We observe the signal disappearance as the molecule is oxidized, Os ${ }^{I I I}$, due to the MLCT electronic resonance absence around 500nm. On the other hand, when the molecule is back to its reduced state, $\mathrm{Os}^{I I}$, the Raman signal is recovered. Inset: Scheme of the Os complex covalently attached to a $600 \mathrm{~nm} \mathrm{Au}$ nanocavity.

to the electronic resonant maximum of PAH-Os described in Fig. 6. Figure 8(bottom) presents a SEM image of one void of the ordered $\mathrm{Au}$ structure, with a scheme of the molecule attached to it (the molecule is of course out of scale). The top panel of Fig. 8 presents Raman spectra taken using $10 \mathrm{~mW}$ of the $514.5 \mathrm{~nm}$ line of an Ar-Kr laser as a function of the electrode potential. The acquisition time was $10 \mathrm{~s}$ and the potential was scanned between $100 \rightarrow 600 \rightarrow 100 \mathrm{mV}$ vs. $\mathrm{Ag} / \mathrm{AgCl}$ at a $10 \mathrm{mV} / \mathrm{s}$ scan rate. To identify the oxidation state corresponding to each spectra the electrochemical potential is given as a function of time at the right of the figure. The peaks correspond to the pyridine stretching vibrations discussed above. The intensity of the $1483 \mathrm{~cm}^{-1}$ peak as a function of time, together with the voltagramm are 
drawn in the bottom panel of Fig. 8. First of all, the excellent signal-to-noise ratio is worth noting, with essentially no additional background, obtained with only $\sim 1 / 20$ of monolayer. Second, it is apparent that the Raman intensity decreases and dissapears completely in the oxidized state $\left(\mathrm{Os}^{I I I}\right)$. Again this can be understood as being due to the shift of the MLCT transition to the UV with the consequent quenching of the electronic resonance. The complete dissapearance of the $1483 \mathrm{~cm}^{-1}$ peak is an indication of good wiring of the Os-complex to the Au substrate. The peaks reappear when the sample returns to the original oxidized state $\left(\mathrm{Os}^{I I}\right)$, signaling the recovery of the Os Raman resonance.

\section{E. flavin electrochemistry in Ag-nanocavities}

In the previous section we have shown how the appropriate tuning of the plasmon modes of ordered cavity arrays can be exploited to enhance electronic resonant Raman signals of Os-complexes allowing the determination of the oxidation state of the redox molecule with sub-monolayer coverage. Such an intensity signal change will be used in the nanobiosensor scheme to be discussed below. In this section we extend these ideas to demonstrate that another biologically relevant redox molecule, namely flavin, can used for the same purpose. In addition, flavin is incorporated as part of glucose oxidase, the enzyme used in the described nanobiosensor, and thus it is important to clearly define which compound is responsible for the observed spectra. Flavines are biological redox molecules that can exchange one or two electrons in aerobic and anaerobic biological media. In glucose oxidase it can efficiently reduce oxygen by 2 -electron reaction to hydrogen peroxide. We will present two-color Raman and in-situ electrochemical studies of flavin assembled on Ag-cavities through a Shiff base, with surface coverages again well below one monolayer.

In the top panel of Fig. 9 we present a voltammogram for methylformylisoalloxazine, a flavin group, covalently attached to a 700nm $\mathrm{Ag}$ nanocavity substrate measured in a Tris buffer at $\mathrm{pH}=8$ between $-100 \rightarrow-700 \rightarrow$ $-100 \mathrm{mV}$ vs. $\mathrm{Ag} / \mathrm{AgCl}$ at $50 \mathrm{mV} / \mathrm{s}$ scan rate. Following the cycle as indicated by the arrow, a negative current peak identifies the reduccion of flavin going through an intermediate semiquinone neutral state. ${ }^{57}$ Continuuing the cycle and decreasing the applied negative potential, a second positive current peak is observed signaling the oxidation of flavin. Optical absorption spectra reported for flavin in solution in its oxidized and neutral semiquinone state show broad $( \pm 100 \mathrm{~nm})$ resonant transitions around $470 \mathrm{~nm}$ and $\sim 600 \mathrm{~nm}$, respectively. ${ }^{58}$ These distinct absorptions allow the possibility to resonantly couple the exciting lasers with them, and at the same time with different plasmons of the $700 \mathrm{~nm} \mathrm{Ag} \mathrm{cavity} \mathrm{with} h \sim 0.7$ which display resonances close to these transitions.

The middle and bottom panels of Fig. 9 present such Raman spectra aquired with in-situ electrochemistry and
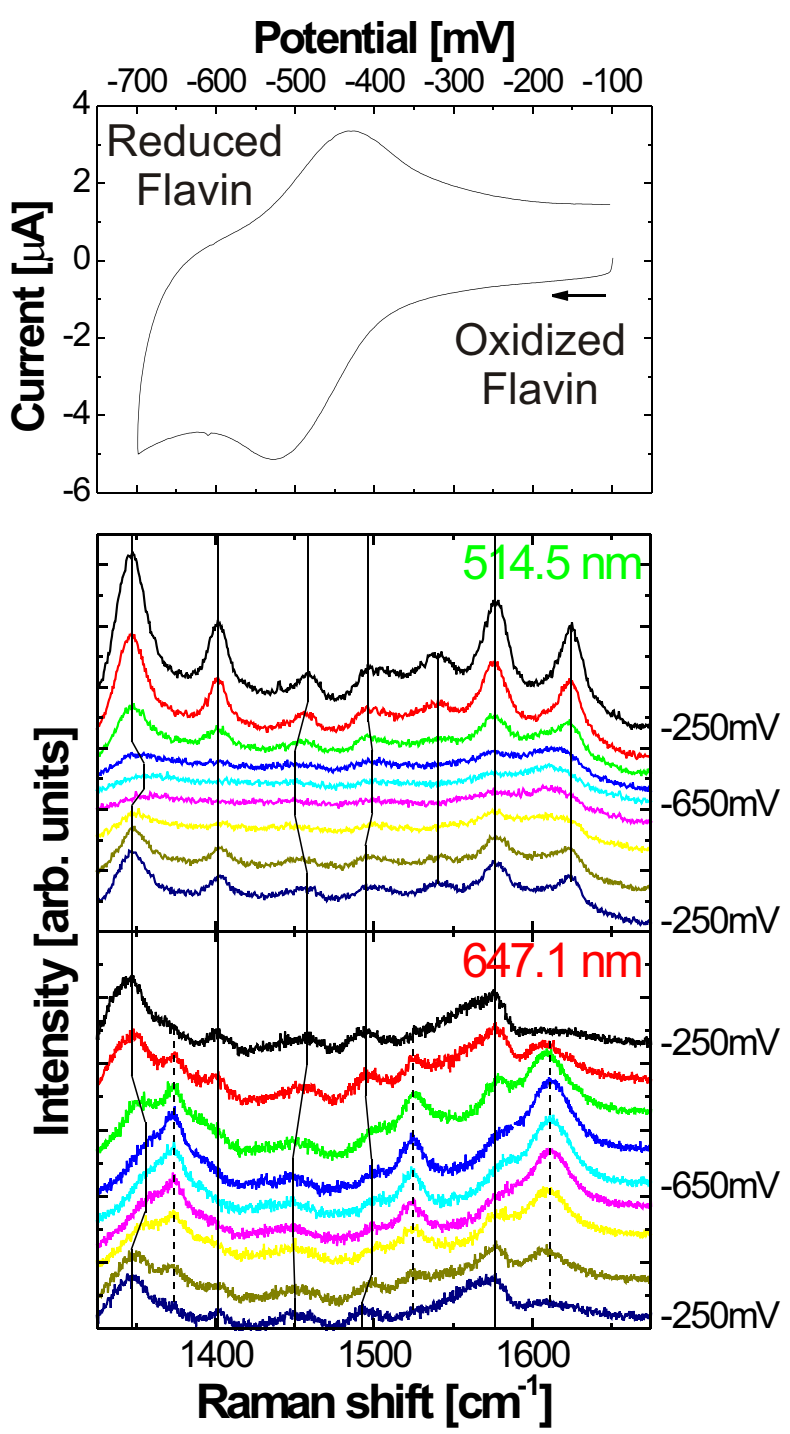

FIG. 9: Top: Voltammogram acquired for methylformylisoalloxazine, a flavin group covalently attached to a $700 \mathrm{~nm} \mathrm{Ag}$ nanocavity substrate and measured in a Tris buffer at $\mathrm{pH}=8$ between $-100 \rightarrow-700 \rightarrow-100 \mathrm{mV}$ vs. $\mathrm{Ag} / \mathrm{AgCl}$ at $50 \mathrm{mV} / \mathrm{s}$ scan rate. Middle (Bottom): Flavin Raman spectra measured as a function of the applied potential using the $514.5 \mathrm{~nm}$ $(647.1 \mathrm{~nm})$ laser line, thus tuning the laser with the electronic resonance of the oxidized (neutral semiquinone) species of Flavin. Vertical lines highlight the main Raman peaks. Full lines indicate those modes that do not shift through the electrochemical experiments. The dotted curves indicate peaks that do shift, while dashed and dashed-dotted lines are used for the Raman peaks that only appear in the upper or lower panel, respectively.

with $514.5 \mathrm{~nm}$ (green) and $647.1 \mathrm{~nm}$ (red) excitation, respectively. We note that in both cases the spectra display strong changes with applied potential, essentially modifying the peak intensity in a way similar to that observed for PAH-Os. Additionally some peaks are ob- 
served to shift with varying oxidation state. What is however most remarkable of the data, is the complementarity of the spectra aquired with 514.5 and $647.1 \mathrm{~nm}$. Peaks that are intense with one laser, are weak with the other, and viceversa. And while the most intense peaks observed with $514.5 \mathrm{~nm}$ dissapear at the most negative applied potentials, the reverse is true for $647.1 \mathrm{~nm}$, for which the most intense peaks appear at negative potentials, but dissapear as the potential is decreased to the oxidized state.

In Fig. 9 the solid lines indicate peaks that belong to the oxidized state of flavin, and reduce their intensity when the molecule changes to the neutral semiquinone form. The dashed lines, on the other hand, highlight those modes that appear in the semiquinone state of the molecule, and are not present in its oxidized form (a tentative assignment of the peaks is provided in the Supplementary Information). Note that with $514.5 \mathrm{~nm}$ excitation the vibrations belonging to the oxidized form are resonantly enhanced, while $647.1 \mathrm{~nm}$ excitation amplifies those belonging to the neutral semiquinone state. These results highlight the rich behavior that can be encountered with redox- molecules, and the different signatures that can be used for sensor applications, i.e., changes in peak intensity, changes in peak position, and contrasting dependencies with excitation wavelength.

\section{F. The core-shell Au glucose nanosensor}

In previous sections we have shown that electronic changes in redox-molecules can be probed by Raman scattering, and that these changes can be sensed efficiently through the use of SERS amplification. In this section we will show how these results can be exploited in biosensors in which the redox molecules are wired to enzymes that specifically recognize target molecules.

We will describe glucose nanosensors built by assembling PAH-Os and GOx multilayers by LbL techniques on Au nanoparticles. Figure 10(top) displays a transmission electron microscopy (TEM) image of the resulting core-shell Au NPs. The image shows the thickness of the glucose oxidase-redox polyelectrolyte shell around the $\mathrm{Au}$ core with a distinct contrast for the gold and the osmium containing soft matter. From the images of many particles we conclude that the Au core dimension is $\sim 20 \mathrm{~nm}$. High resolution transmission electron microscopy (HRTEM) observations also confirmed that the $\mathrm{Au}$ cores are crystalline. The thickness of the multilayer shell for three enzyme and four polyelectrolyte layers was found to be $\sim 19 \mathrm{~nm} .^{8}$ Figure 10 also presents schematically the working principle of the proposed glucose sensor. The PAH-Os complex is previously chemically prepared in its oxidized (Raman "silent") state. On contact with glucose, charge is transferred to GOx converting glucose into gluconic acid. Through efficient wiring with the redox-molecule, charge is then passed to PAH-Os reducing it to its Os(II) (Raman "active") state. The intensity

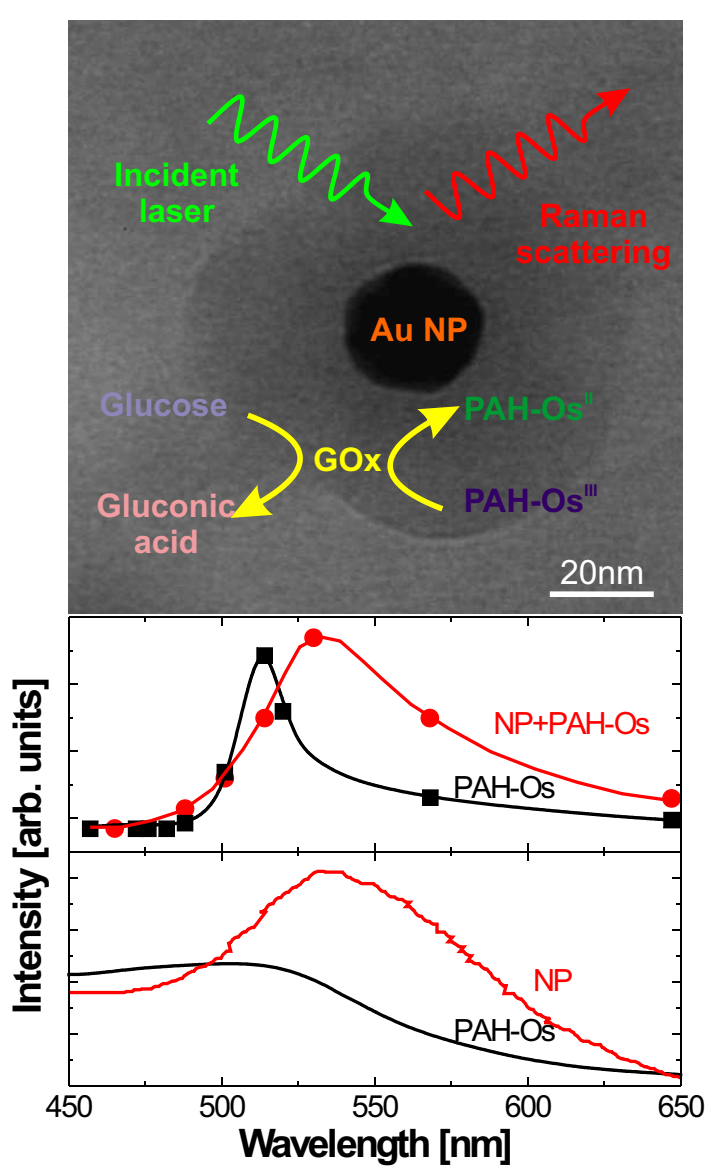

FIG. 10: Top: Schematic representation of the resonant Raman interrogation of the Os(II) concentration in core-shell $\mathrm{Au}$ NPs and glucose detection. The background picture corresponds to the TEM image of a (PAH-Os/GOx $)_{3}$ core shell $\mathrm{Au}$ NP. Middle: Raman intensity of the $383 \mathrm{~cm}^{-1}$ peak for the osmium complex in PAH-Os as a function of the laser excitation energy both for the complex assembled in a layerby-layer grown supramolecular structure of (PAH-Os/PVS $)_{n}$ $(n=7)$ grown on a glass substrate (i.e., without SERS effect) and in the core-shell nanosensor. Bottom: Detail of the absorption spectra of the PAH-Os complex and of the used $\mathrm{Au}-\mathrm{NPs}$ in the spectral region corresponding to the metalto-ligand charge transfer (MLCT) transition. Note the close correspondence of the absorption maxima of the Os complex and the NP plasmon, with the resonant Raman maxima for the pure complex and the core-shell sensors, respectively.

recovery of the PAH-Os Raman signals when Os(III) is reduced to Os(II) is proportional to the concentration of glucose. The Au nanoparticle plays the role of providing the required substrate, and at the same time amplifying the SERS response which can be interrogated remotely.

Figure 10(bottom) presents a detail of the absorption peaks of both the PAH-Os complex (black) and the $\mathrm{Au}$ NPs (red) in the spectral region around the MLCT transition of the redox-molecule. Due to the spectral overlap of the two resonances it is apparent that a plasmon-induced 
surface enhancement of the Raman signal should add to the electronic resonance described above to provide a particularly sensitive Raman probe. To evidence the presence of SERS amplification we present in the same figure a comparison of the resonant Raman response of the PAH-Os complex in the LbL supramolecular structure (PAH-Os $)_{4} /(\mathrm{PVS})_{3}$ grown on a glass substrate (i.e., without SERS effect), with that of the core-shell nanosensor. Figure 10(middle) shows the Raman intensity of the pyridine-stretching $1550 \mathrm{~cm}^{-1}$ mode of the osmium complex as a function of the laser excitation energy. For $\mathrm{PAH}-\mathrm{Os}$ in the $(\mathrm{PAH}-\mathrm{Os})_{4} /(\mathrm{PVS})_{3} \mathrm{ML}$ grown on glass we see the electronic Raman resonances at $\sim 515 \mathrm{~nm}$ due to the energy tuning between the incident photon and the osmium pyridine-bipyridine complex MCTL transition already discussed in Sect. IIIB. The Raman scattering for the osmium complex in the multilayer structure adsorbed in the core-shell Au nanoparticles shows a quite distinct profile as a function of laser excitation energy (labeled NP+PAH-Os in Fig. 10(middle)). The resonance maximum shifts to lower energies $(\sim 530 \mathrm{~nm})$, in coincidence with the plasmon resonance maximum, and a much broader response is observed. Unlike the electronic resonant Raman scattering of the PAHOs alone, on core-shell NPs the intensity of the bipyridine Raman scattering extends over the $550-650 \mathrm{~nm}$ region following the Au plasmon absorption profile.

Our strategy for this specific contactless glucose sensor has been to recover the resonant Raman signal due to the Os(II) complex in the NPs shell via the enzymatic reduction of the Raman silent Os(III) complex in the presence of glucose and catalyzed by glucose oxidase in the NPs shell. As described in the experimental section, to obtain the oxidized nanoparticles, Os(II) in the shell was oxidized by treatment with $\mathrm{H}_{2} \mathrm{O}_{2}$ catalyzed by soluble soybean peroxidase (SBP), followed by thorough washing and centrifugation steps.

Figure 11 (left) shows Raman spectra collected with $10 \mathrm{~mW}$ of $514.5 \mathrm{~nm}$ excitation and $10 \mathrm{~s}$ acquisition times. The spectra were taken from $1 \mu \mathrm{L}$ drops of the core-shell NPs solution on atomically flat Au coated glass substrate (robax, Arandee, Germany) and prepared (i) as grown, that is, in its reduced Os(II) state, (ii) oxidized to Os(III), and (iii) reduced with glucose added to the oxidized sensor nanoparticles. The PAH-Os bypiridinestretching modes are present with good signal-to-noise ratio in the original core-shell NPs with Os in its reduced state (labeled "as-grown"). In the oxidized state, Os(III), the Raman peaks dissapear completely. The signal, however, recovers to $20 \%$ of the original Os(II) NPs spectrum when the oxidized NPs are put into contact with glucose. For this purpose $5 \mu \mathrm{L}$ of $1 \mathrm{M}$ glucose solution were added to $25 \mu \mathrm{L}$ of solution of the oxidized nanoparticles, with a washing cycle of $35 \mathrm{~min}$ at $7000 \mathrm{rpm}(4.500 \mathrm{~g})$ to remove excess glucose.

The recovery of the Os(II) resonant Raman intensity demonstrates the possibility of developing a photonic nanobiosensor using the core-shell nanoparticles. As dis-

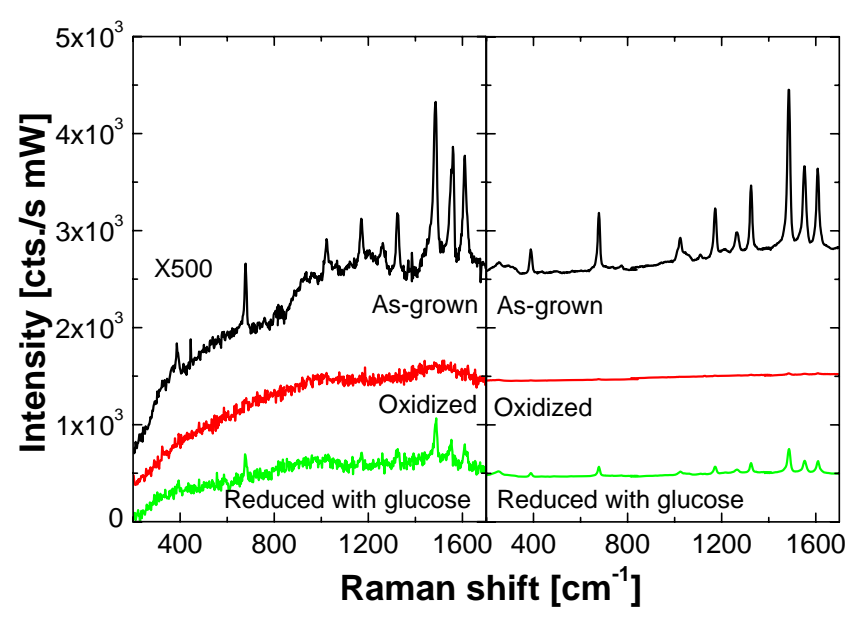

FIG. 11: Left: Resonant Raman spectra of Au core-shell PAH-Os+(GOx/PAH-Os $)_{3}$ nanoparticles deposited on an atomically flat Au substrate for (i) as grown Os(II), (ii) oxidized Os(III), and (iii) after addition of glucose solution (200 $\mathrm{mM})$. The spectra were acquired with $10 \mathrm{~mW} 514.5 \mathrm{~nm}$ excitation and $10 \mathrm{~s}$ acquisition time. Right: Same sequence of Raman spectra but with the core-shell NPs deposited on 700 $\mathrm{nm} \mathrm{Ag}$ cavities. Note the $\times 500$ enhancement of the signals, with otherwise the same relation between spectra from samples reduced, oxidized, and in contact with glucose.

cussed above, the spectra shown in Fig. 11 (left) are simultaneously enhanced by electronic and plasmon resonance effects, an amplification property that is intrinsic to the proposed nanosensor. It should be noticed however that, in this series of experiments where a $1 \mu \mathrm{L}$ drop of the NPs solution was placed on a flat Au surface under the laser spot, no surface Raman enhancement is expected due to the gold surface. On the other hand, the contact of the core-shell sensors with glucose can be sensed ex-situ exploiting the additional resonant enhancement of appropriately designed SERS substrates, as the nanocavities presented in Sect. III D. ${ }^{59}$ To illustrate this we show in Fig. 11 (right) spectra collected with the same sequence of samples (i-iii) presented in Fig. 11 (left), but now with the Au core-shell multilayer nanoparticles deposited and dried on a $700 \mathrm{~nm}$ diameter $\mathrm{Ag}$ ordered cavity-array similar to that used with flavin in Sect. IIIE. It is apparent that a further $\times 500$ enhancement of the signal is obtained when using the Ag nanostructured cavity surface with a much better signalto-noise ratio. The overall sensor response (i.e., oxidized vs reduced Os signal, and recovery after contact with glucose), however, remains unaltered, furthermore confirming its potentiality for analytical applications.

As a proof of principle we studied the nanosensor response on glucose concentration. Figure 12 presents the dependence of the Raman signal ((1480 cm-1 mode intensity in counts per second per $\mathrm{mW}$ of $514.5 \mathrm{~nm}$ laser power) as seen in a typical calibration curve. The back- 


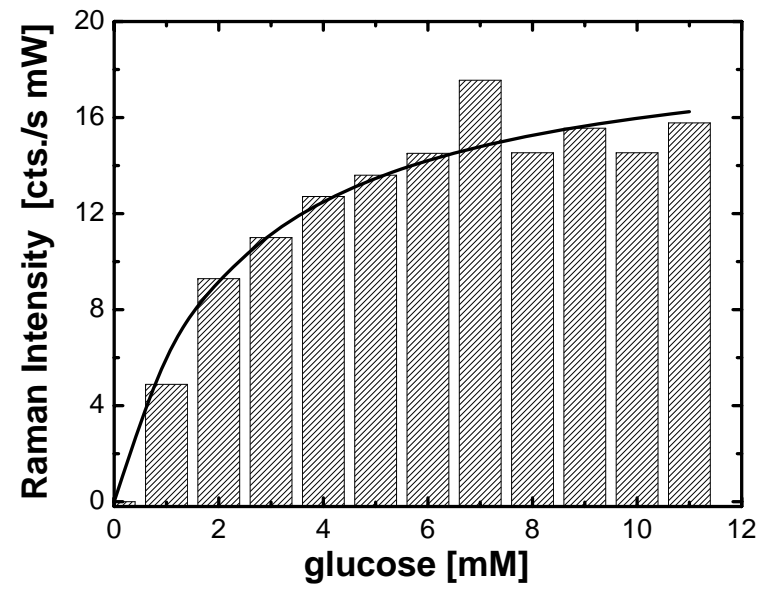

FIG. 12: Resonant Raman scattering intensity for the Os(II) vibration mode at $1480 \mathrm{~cm}-1$ vs glucose concentration. The data were obtained with $1 \mu \mathrm{L}$ drops of aqueous solution of $60 \mathrm{pM}$ Au core (PAH-Os $)_{4}(\mathrm{GOx})_{3}$ shell nanoparticles on 700 $\mathrm{nm} \mathrm{Ag}$ cavities. The continuous curve is a fit to a MichaelisMenten type equation.

ground signal of the Os(III) NPs solution in the absence of glucose has been substracted. To collect these data a $1 \mu \mathrm{L}$ drop of solution containing the NPs and glucose was placed on a nanostructured Ag surface, and the Raman scattering was measured immediately to avoid any evaporation of the solution. Each point was measured with a fresh solution of nanoparticles and glucose, keeping the concentration of NPs constant $(60.5 \mathrm{pM})$ while varying the glucose concentration. We estimate the number of NPs within the probed volume below 1000. Note also that, in contrast with the data in Fig. 11, the drop was not allowed to dry and thus only a small fraction of the NPs is amplified by the cavity SERS effect. The Raman intensity increases with the amount of glucose, reaching saturation at $7-8 \mathrm{mM}$ glucose. Good fit of the experimental data to a Michaelis-Menten type equation is observed. ${ }^{60}$ The results shown in Figure 8 indicate that the resonant Raman scattering intensity grows with the glucose concentration following the rate of glucose enzymatic oxidation and therefore the rate of Os(II) formation.

\section{CONCLUSIONS}

We presented a complete study of the optical properties of a a glucose nanosensor based on the enzymatic recognition of the target molecule, the consequent reduction of a PAH-Os "wire" interrogated contactlessly through Raman scattering, and the SERS amplification using appropriately tailored SERS substrates. The different building blocks of the sensor were characterized and studied separately. To begin with, the properties of (PAH-Os/PVS $)_{n}$ self-assembled multilayers were analyzed structurally through variable angle spectroscopic ellipsometry. This investigation showed that a multisample analysis can be used to extract thickness information of ultra-thin organic multilayers, providing in addition the optical constant ( $n$ and $k$ ) of the films which are valuable data to characterize the electronic resonance of the PAH-Os redox-molecule. This information is relevant to understand the electronic resonant Raman response of the Os-complex, which is shown to display strong enhancement with laser excitation around $\sim 510 \mathrm{~nm}$. This enhancement is quenched upon oxidation of the Oscomplex to Os(III), in agreement with absorption spectra in solution which indicate that the MLCT transition responsible for the Raman resonance shifts to the UV after oxidation with ceric ions. The oxidation-state dependence of the Raman peak intensity is proposed as active principle for the optically interrogable glucose nanosensor.

We show that essentially the same behavior can be reproduced with the Os-complex wired to the substrate through a covalent bond, and using the biologically relevant flavin as alternative redox-molecule. In the latter case we observe a rich behavior that provides additional signatures potentially interesting for sensing purposes, i.e., shifts of peaks, and laser-wavelength sensitivity to different oxidation states of the molecule. In these investigations, strong and well defined Raman signals were demonstrated with in-situ electrochemical experiments with sub-monolayer molecular surface coverages using metallic Au and Ag ordered cavity arrays as efficient and spectrally tunable SERS substrates.

These building blocks, redox-molecule sensing, and SERS enhancement, were then put together with an enzyme to achieve a fully integrated core-shell nanoparticle system selectively responsive to glucose. The system is comprised of LbL assembled GOx/PAH-Os multilayers on core-shell $\mathrm{Au}$ nanoparticles. The response to glucose concentration is based on the different resonant Raman behavior of the Os(II) and Os(III) polymer molecular wire. Contactless sensing through the Os(II) resonant Raman signal has been demonstrated. Amplification by the $\mathrm{Au}$ NPs plasmon resonance has also been evidenced leading to an extra enhancement of the biosensor signal. Furthermore, by placing the glucose nanosensors on $\mathrm{Ag}$ nanocavity array substrate we demonstrated a 500 extra amplification factor which allows us to increase the analytical capabilities of these sensors. It should be emphasized that all the components for the selective molecular recognition and the generation of an optical signal: the osmium molecular wire, the glucose oxidase, and the SERS amplifier, are self-contained in each nanoparticle biosensor. Similar schemes could be applied to SERS substrates other than NPs for in-situ and ex-situ sensing. In the latter case, e.g. using the described nanocavity SERS substrates, electrochemical sensing and erasing could be added to the Raman interrogation providing further versatility to the sensor. 


\section{ACKNOWLEDGEMENTS}

The authors are grateful to CONICET, University of Buenos Aires, and CNEA for financial support. ANPCyT (BID 1728 OC-AR PICT 2003-17170, and PICT 2006-01061) and the Argentine Nanotechnology Network PAV 2004 Project 22711- BID 1728/OC-AR grants are acknowledged. P.D.S. acknowledges a research doctoral fellowship from CONICET. E.J.C., A.F., and N. G. T., are permanent fellows of CONICET, Argentina. We thank Prof. Phil Bartlett for a short stay of N. Tognalli at Southampton to learn the template nanostructure techniques under Welcome Trust, U.K. (Grant 69713). H. Troiani is deeply acknowledged for the TEM images of the core-shell nanosensors, and C. Bonazzola for the preparation of some of the samples.
† e-mail: afains@cab.cnea.gov.ar

${ }^{1}$ Decher, G. Science, 277, 1232 (1997).

${ }^{2}$ Lvov, Y.; Ariga, K; Ichinose, I.; Kunitake, T. J. Am. Chem. Soc., 117, 6117 (1995).

3 J. B. Schlenoff, in Multilayer Thin Films: Segmental assembly of nanocomposite materials, eds. Decher, G. and Schlenoff, J. B. (Wiley, New York, 2003).

${ }^{4}$ Hodak, J.; Etchenique, R.; Calvo, E. J.; Singhal, K.; Bartlett, P. N. Langmuir, 13, 2708 (1997).

${ }^{5}$ Calvo, E. J.; Etchenique, R.; Pietrasanta, L.; Wolosiuk, A.; Danilowicz, C. Anal. Chem., 73, 1161 (2001).

${ }^{6}$ Calvo, E. J.; Battaglini, F.; Danilowicz, C.; Wolosiuk, A.; Otero, M. Faraday Discuss., 47 (2000).

7 Calvo, E. J.; Danilowicz, C.; Wolosiuk, A. J. Am. Chem. Soc., 124, 2452 (2002).

8 Flexer, V.; Forzani, E. S.; Calvo, E. J. Anal. Chem., 78, 399 (2006).

${ }^{9}$ Bonazzola, C.; Calvo, E. J.; Nart, F. C. Langmuir, 19, 5279 (2003)

10 Tognalli, N.; Fainstein, A.; Bonazzola, C.; Calvo, E. J. J. Chem. Phys., 120, 1905 (2004).

11 Caruso, F. Adv. Mater., 13, 11 (2001).

12 Caruso, F.; Caruso, R. A.; Mohwald, H. Science, 282, 1111 (1998).

13 Gittins, D. I.; Caruso, F. Adv. Mater., 12, 1947 (2000).

${ }^{14}$ Gittins, D. I.; Caruso, F. J. Phys. Chem. B, 105, 6846 (2001).

15 Elghanian, R.; Storhoff, J. J.; Mucic, R. C.; Letsinger, R. L.; Mirkin, C. A. Science, 277, 1078 (1997).

${ }^{16}$ Maxwell, D. J.; Taylor, J. R.; Nie, S. M. J. Am. Chem. Soc., 124, 9606 (2002).

17 Willner, I.; Willner, B.; Katz, E. Bioelectrochemistry, 70, 2 (2007)

18 He, L; Musick, M. D.; Nicewarner, S. R.; Salinas, F. G.; Benkovic, S. J.; Natan, M. J.; Keating, C. D. J. Am. Chem. Soc., 122, 9071 (2000).

19 Perez, J. M.; Simeone, F. J.; Tsourkas, A.; Josephson, L.; Weissleder, R. Nano Lett., 4, 119 (2004).

${ }^{20}$ Doering, W. E.; Piotti, M. E.; Natan, M. J. Adv. Mater., 19, 3100 (2007)

${ }^{21}$ Qian, X. M.; Peng, X. H.; Ansari, D. O.; Yin-Goen, Q.; Chen, G. Z.; Shin, D. M.; Yang, L.; Young, A. N.; Wang, M. D.; Nie, S. Nat. Biotechnol., 26, 83 (2008).

22 Willets, K. A.; Van Duyne, R. P. Annu. ReV. Phys. Chem., 58, 267 (2007).

23 Etchegoin, P.; Maher, R. C.; Cohen, L. F.; Hartigan H.; Brown, R. J. C.; Milton, M. J. T.; Gallop, J. C. Chem. Phys. Lett., 375, 84 (2003).

${ }^{24}$ Freeman, R. G.; Grabar, K. C.; Allison, K. J.; Bright, R. M.; Davis, J. A.; Guthrie, A. P.; Hommer, M. B.; Jackson, M. A.; Smith, P. C.; Walter, D. G.; Natan, M. J. Science,
267, 1629 (1995).

${ }^{25}$ Kiely, C. J.; Fink, J.; Brust, M.; Bethell, D.; Schiffrin, D. J. Nature, 396, 444 (1998).

${ }^{26}$ Félidj, N.; Aubard, J.; Lévi, G.; Krenn, J. R.; Salerno, M.; Schider, G.; Lamprecht, B.; Leitner, A.; Aussenegg, F. R. Phys. Rev. B, 65, 075419 (2002).

27 Jackson, J. B.; Westcott, S. L.; Hirsch, L. R.; West, J. L.; Halas, N. J. Appl. Phys. Lett., 82, 257 (2003).

${ }^{28}$ Kelly, K. L.; Coronado, E.; Zhao, L. L.; Schatz, G. C. J. Phys. Chem. B, 107, 668 (2003).

29 Dick, L. A.; McFarland, A. D.; Haynes, C. L.; Van Duyne, R. P. J. Phys. Chem. B, 106, 853 (2002).

${ }^{30}$ Le Ru, E. C.; Meyer, M.; Etchegoin, P. J. Phys. Chem. B, 110, 1944 (2006).

31 Maher, R. C.; Cohen, L. F.; Etchegoin, P. Chem. Phys. Lett., 352, 378 (2002).

32 Tognalli, N.; Fainstein, A.; Calvo, E.; Bonazzola, C.; Pietrasanta, L.; Campoy-Quiles, M.; Etchegoin, P. J. Chem. Phys., 123044707 (2005).

33 Bartlett, P. N.; Birkin, P. R.; Ghanem, M. A. Chem. Comm., 1671 (2000).

${ }^{34}$ Coyle, S.; Netti, M. C.; Baumberg, J. J.; Ghanem, M. A., Birkin, P. R.; Bartlett, P. N.; Whittaker, D. M. Phys. Rev. Lett., 87, 176801 (2001).

${ }^{35}$ Kelf, T. A.; Sugawara, Y.; Baumberg, J. J.; Abdelsalam, M. E.; Bartlett, P. N. Phys. Rev. Lett., 95, 116802 (2005).

${ }^{36}$ Kelf, T. A.; Sugawara, Y.; Cole, R. M.; Baumberg, J. J.; Abdelsalam, M. E.; Cintra, S.; Mahajan, S.; Russell, A. E.; Bartlett, P. N. Phys. Rev. B, 74, 245415 (2006).

37 Cole, R. M.; Baumberg, J. J.; García de Abajo, F. J.; Mahajan, S.; Abdelsalam, M. E.; Bartlett, P. N. Nano Lett., 7, 2094 (2007).

38 Teperik, T. V.; Popov, V. V.; García de Abajo, F. J.; Abdelsalam, M. Bartlett, P. N. Kelf, T. A.; Sugawara, Y.; Baumberg, J. J. Optics Express, 14, 1965 (2006).

39 Lacharmoise, P. D.; Tognalli, N. G.; Goñi, A. R.; Alonso, M. I.; Fainstein, A.; Cole, R. M.; Baumberg, J. J.; García de Abajo, F. J.; Bartlett, P. N. Phys. Rev. B, 78, 125410 (2008).

40 Baumberg, J. J.; Kelf, T. A.; Sugawara, Y.; Cintra, S.; Abdelsalam, M. E.; Bartlett, P. N.; Russell, A. Nano Lett., 5, $2262(2005)$.

41 Cintra, S. H.; Abdelsalam, M. E.; Bartlett, P. N.; Baumberg, J. J.; Kelf, T. A.; Sugawara, Y.; Russell, A. E. Faraday Discuss., 132, 191 (2006).

42 Calvo, E. J.; Battaglini, F.; Danilowicz, C.; Wolosiuk, A.; Otero, M. Faraday Discuss., 116, 47 (2000).

43 Forzani, E. S.; Otero, M.; Pérez, M. A.; López Teijelo, M.; Calvo, E. J. Langmuir, 18, 4020 (2002).

44 Ricci, A; Rolli, C; Rothacher, S; Baraldo, L; Bonazzola, C; Calvo, E. J.; Tognalli, N.; Fainstein, A., J. Solid State 
Electrochem., 11, 1511 (2007).

${ }^{45}$ Calvo, E. J.; Rothacher, M. S.; Bonazzola, C.; Wheeldon, I. R.; Salvarezza, R. C.; Vela, M. E.; Benitez, G. Langmuir, 21, 7907 (2005).

46 Bonazzola, C.; Brust, M.; Calvo, E. J. J. Electroanal. Chem., 407, 203 (1996).

47 Abdelsalam, M; Bartlett, P. N.; Russell, A. E.; Baumberg, J. J.; Calvo, E. J.; Tognalli, N. G.; Fainstein, A. Langmuir, 24, 7018 (2008).

48 Scodeller, P.; Flexer, V.; Szamocki, R.; Calvo, E. J.; Tognalli, N.; Troiani, H.; Fainstein, A. J. Am. Chem. Soc., 130, 12690 (2008)

49 Tompkins, H. G.; McGahan, W. A. Spectroscopic ellipsometry and reflectometry, John Wiley \& sons, Inc., Toronto (1999).

50 Campoy-Quiles, M. Ellipsometry applied to Organic Materials for Optoelectronic and Photonic Applications. Transfer Report Essay, Imperial College London (2003).

51 Taylor, A.P.; Crayston, J.A.; Dines, T. J. J. Chem. Soc. Faraday Trans., 93, 1803 (1997).

${ }^{52}$ Walsh, D. A.; Keyes, T. E.; Hogan, C. F.; Forster, R. J. J.
Phys. Chem. B, 15, 2792 (2001).

53 Winkler, E.; Etchegoin, P.; Fainstein, A.; Fainstein,C. Phys. Rev. B, 57, 13477 (1998).

${ }^{54}$ Winkler, E.; Fainstein, A.; Etchegoin, P.; Fainstein, C. Phys. Rev. B, 61, 15756 (2000).

${ }^{55}$ Le Ru, E. C.; Etchegoin, P. G. Principles of surface enhanced Raman spectroscopy and related plasmonic effects. Elsevier, Amsterdam (2008).

56 Mie, G. Ann. Phys., 25, 377 (1908).

57 Xu, J.; Birke, R. L.; Lombardi, J. R. J. Am. Chem. Soc., 1095646 (1987).

58 Ghisla, S.; Massey, V.; Lhoste, J. M.; Mayhew, S. G. Biochemistry, 13589 (1974).

59 Abdelsalam, M.; Bartlett, P. N.; Russell, A. E.; Baumberg, J. J.; Calvo, E. J.; Tognalli, N.; Fainstein, A. Langmuir, 24, 7018 (2008).

${ }^{60}$ Calvo, E. J.; Flexer, V.; Toh, C. S.; Bartlett, P. N. in Bioelectrochemistry: fundamentals, experimental techniques and applications, Bartlett, P. N. Ed., John Wiley \& Sons (2008). 